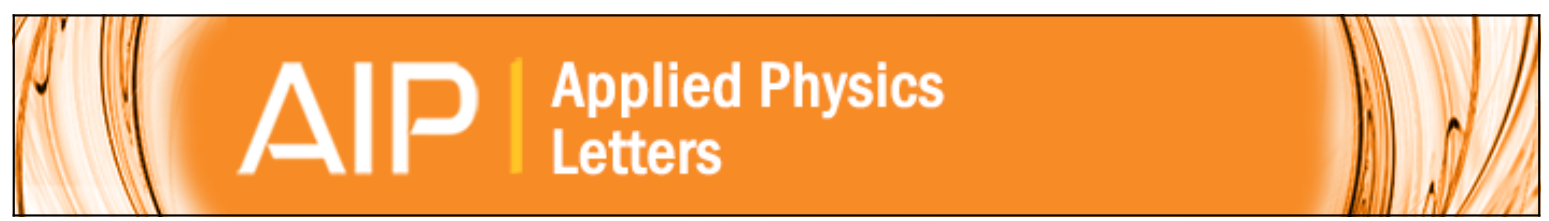

\title{
Realistic adsorption geometries and binding affinities of metal nanoparticles onto the surface of carbon nanotubes
}

Noejung Park, Dongchul Sung, Seokho Lim, Seongho Moon, and Suklyun Hong

Citation: Applied Physics Letters 94, 073105 (2009); doi: 10.1063/1.3083548

View online: $\mathrm{http}: / / \mathrm{dx}$. doi.org/10.1063/1.3083548

View Table of Contents: http://scitation.aip.org/content/aip/journal/apl/94/7?ver=pdfcov

Published by the AIP Publishing

\section{Articles you may be interested in}

Insight into the description of van der Waals forces for benzene adsorption on transition metal (111) surfaces

J. Chem. Phys. 140, 084704 (2014); 10.1063/1.4866175

The role of van der Waals forces in water adsorption on metals

J. Chem. Phys. 138, 024708 (2013); 10.1063/1.4773901

Coating geometries of metals on single-walled carbon nanotubes

Appl. Phys. Lett. 96, 063108 (2010); 10.1063/1.3309752

Adsorption of transition-metal atoms on boron nitride nanotube: A density-functional study

J. Chem. Phys. 125, 044711 (2006); 10.1063/1.2218841

Binding at molecule/gold transport interfaces. V. Comparison of different metals and molecular bridges

J. Chem. Phys. 123, 234704 (2005); 10.1063/1.1947747

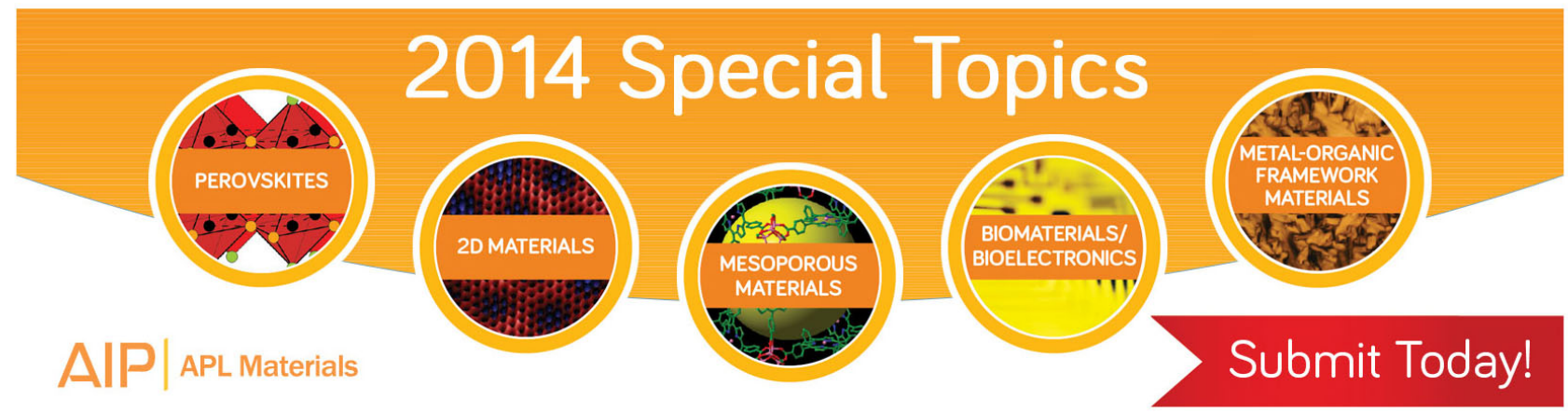




\title{
Realistic adsorption geometries and binding affinities of metal nanoparticles onto the surface of carbon nanotubes
}

\author{
Noejung Park ${ }^{1, a), b)}$ Dongchul Sung, ${ }^{2,3}$ Seokho Lim, ${ }^{1}$ Seongho Moon, ${ }^{4}$ and \\ Suklyun Hong ${ }^{2, a)}$ \\ ${ }^{1}$ Department of Applied Physics, Dankook University, Yongin 448-701, Republic of Korea \\ ${ }^{2}$ Department of Physics and Institute of Fundamental Physics, Sejong University, Seoul 143-747, \\ Republic of Korea \\ ${ }^{3}$ Department of Physics, Korea University, Seoul 136-713, Republic of Korea \\ ${ }^{4}$ Samsung Electronics Co., Ltd., Yongin 446-711, Republic of Korea
}

(Received 20 November 2008; accepted 27 January 2009; published online 17 February 2009)

\begin{abstract}
Adsorption geometries and binding affinities of metal nanoparticles onto carbon nanotubes (CNTs) are investigated through density-functional-theory calculations. Clusters of 13 metal atoms are used as models for metal nanoparticles. Palladium, platinum, and titanium particles strongly chemisorb to the CNT surface. Unlike the cases of atomic adsorptions the aluminum particle has the weakest binding affinity with the CNT. Aluminum or gold nanoparticles accumulated on the CNT develop the triangular bonding network of the metal surfaces in which the metal-carbon bond is not favored. This suggests that the CNT-Al interface is likely to have many voids and thus susceptible to oxidation damages. () 2009 American Institute of Physics. [DOI: 10.1063/1.3083548]
\end{abstract}

Carbon nanotubes (CNTs) have been the significant focus of scientific research during the past few decades. Studies of the electron system in nearly one-dimensional geometry have revealed various intriguing quantum mechanical phenomena, which have stimulated not only academic interest but ideas for potential applications. ${ }^{1,2}$ In laboratories various device patterns, including field effect transistors (FETs), ${ }^{3}$ electron field emitters, ${ }^{4}$ and electromechanical switches, ${ }^{5}$ have been fabricated. In particular, the maximum current density through an individual CNT is known to be three orders of magnitude larger than that of $\mathrm{Cu}$ wire, making CNTs very promising for use in hole interconnect. ${ }^{6,7}$ For those applications, the metal-CNT interface may constitute the central part of study because of the significance of contact resistance. On the other hand, the metal-CNT binding strength has been questioned in view of composite material. ${ }^{8}$ Previous studies investigated wetting properties of metal nanoparticles on the surface of CNTs. ${ }^{9,10}$ Throughout experiments it has been generally noticed that $\mathrm{Au}, \mathrm{Al}, \mathrm{Fe}, \mathrm{Pb}$, and $\mathrm{Zn}$ have poor wetting onto the surface of CNTs. Meanwhile, it has been suggested that the Al-CNT interfaces are particularly liable to contamination by environmental oxygen species. ${ }^{11,12}$ Such experimental results are not well supported by theoretical studies. Theorists have reported that the adsorption of $\mathrm{Al}$ atom onto CNTs and the adhesion of CNTs to the Al surface are decently strong. ${ }^{13,14}$ Besides the cases of $\mathrm{Al}$, understanding of the interfacial structure between CNT and other metals was not sufficient in previous studies, even though such knowledge has been necessary for an analysis of the realistic Schottky barrier. ${ }^{15-17}$

The objective of the present work is to investigate a realistic interfacial geometry between the CNT and various metals. In actual device fabrication process, evaporated metal particles are to be deposited onto CNTs. Regarding

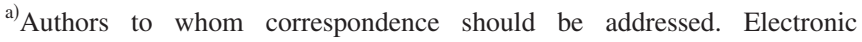
addresses: noejung@dku.edu and hong@sejong.ac.kr.

${ }^{b)}$ FAX: +82-31-8005-3208.
}

such process, the adsorption with individual metal atoms onto CNTs or the adhesion of CNTs to a well-developed flat surface of bulk metals may not be a right choice for the model. We choose the metal nanoparticles consisting of 13 atoms $\left(M_{13}\right)$ as the model. Note that the $M_{13}$ can constitute the minimal cluster of closed atomic shell, and thus its adsorption geometry on the surface of the CNT could be an indicator of the wetting property of metal nanoparticles. ${ }^{18}$ Our results in the present work show that the $\mathrm{Al}$ and $\mathrm{Au}$ nanoparticles spontaneously form a (111)-like surface reconstruction in which the bonding to the CNT surface becomes unfavorable. In contrast, $\mathrm{Pt}, \mathrm{Pd}$, and Ti have strong binding to the CNT. Our calculations are based on the total energy calculation within the density functional theory. ${ }^{19}$ Throughout the present work, we use the Vienna ab initio Simulation Package and the provided pseudopotentials. ${ }^{20,21}$ The generalized gradient approximation (GGA) and the local density approximation (LDA) ${ }^{22,23}$ are used in the description of the exchange correlation potential. The plane-wave basis set is employed with the energy cutoff of $400 \mathrm{eV}^{24}$

Figures 1(a)-1(e) are the GGA optimized geometries of the $(5,5) \mathrm{CNT}$ with the adsorbed $\mathrm{Al}_{13}, \mathrm{Au}_{13}, \mathrm{Pd}_{13}, \mathrm{Pt}_{13}$, and $\mathrm{Ti}_{13}$. The selected low energy structures of $\mathrm{Au}_{13}$ (flake), $\mathrm{Ti}_{13}$ (icosahedron), and $\mathrm{Pt}_{13}$ (cage) are shown in Fig. 1(f). In order to find the lowest energy structure of $M_{13}$ particles, the suggestions in previous works are referenced and recalculated independently. We found that the $\mathrm{Al}_{13}$ is close to the regular icosahedron, irrespective of the chosen density functional. ${ }^{25}$ For $\mathrm{Au}_{13}$, we confirmed that the flake geometry has lower energy than the cuboctahedron by $1.4 \mathrm{eV}$ in both the LDA and GGA calculations. ${ }^{26}$ Our full $a b$ initio calculations of $\mathrm{Pt}_{13}$ resulted in a few low energy cage structures: ${ }^{27}$ the lowest energy structure is shown in Fig. 1(f). For the case of $\mathrm{Pd}_{13}$ the icosahedron constitutes the low energy isomers along with a few cage structures. Both the LDA and GGA calculations resulted in the icosahedral $\mathrm{Ti}_{13}$ with the $D_{3 d}$ symmetry in which one of the triangles is slightly larger than others. ${ }^{28}$ 
(a)

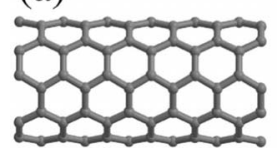

(d)

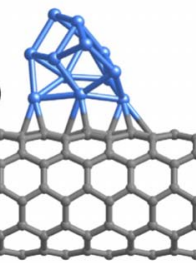

(b)

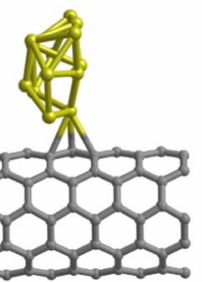

(e)
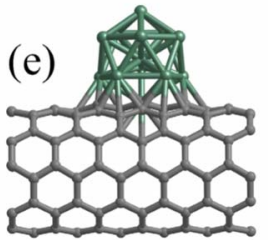

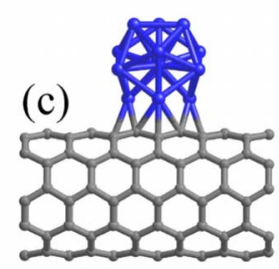

(f)

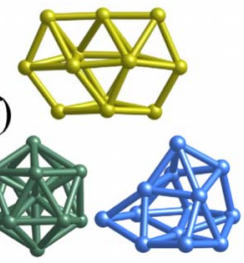

FIG. 1. (Color online) Optimized geometries for (a) $\mathrm{Al}_{13}$, (b) $\mathrm{Au}_{13}$, (c) $\mathrm{Pd}_{13}$, (d) $\mathrm{Pt}_{13}$, and (e) $\mathrm{Ti}_{13}$ onto the $(5,5) \mathrm{CNT}$. (f) Optimized geometries of the flake-type $\mathrm{Au}_{13}$, icosahedral $\mathrm{Ti}_{13}$, and cage-type $\mathrm{Pt}_{13}$. Throughout (a) to (e) the metal-carbon distance less than $2.5 \AA$ is drawn with bond lines. This bond line rule also applies to Figs. 2 and 3.

In order to search for the adsorption geometries, we performed the geometry optimization with various initial conditions: different orientations of the $M_{13}$ particle on different binding sites of the surface of the CNT. The obtained lowest energy adsorption geometries are shown in Fig. 1. Among them the $\mathrm{Al}_{13}$ has the weakest binding affinity with the CNT and preserves the icosahedron geometry of the isolated cluster, in consistency with the previous work. ${ }^{29}$ The binding energies of the $M_{13}$ particles onto the CNT were calculated as follows: $E_{b}=-\left[E_{\mathrm{tot}}\left(M_{13} / \mathrm{CNT}\right)-E_{\mathrm{tot}}\left(M_{13}\right)-E_{\mathrm{tot}}(\mathrm{CNT})\right]$, where the $E_{\text {tot }}$ represents the total energy of the optimized geometry for each case. The results of binding energies are summarized in Table I. Despite our extensive search for the lowest energy adsorption geometry, we cannot exclude the possibility that some unidentified structures might have lower energies than those shown in Fig. 1. Therefore, the energetics shown in Table I should be interpreted with care. However, the overall trend in Table I undoubtedly indicates that $\mathrm{Pt}, \mathrm{Pd}$, and Ti have strong binding to the surface of CNT, whereas $\mathrm{Al}$ and $\mathrm{Au}$ have a weak binding affinity. The strong binding natures of $\mathrm{Pt}, \mathrm{Pd}$, and Ti particles onto the CNT are consistent with the known chemical reactivities of the metal surfaces. ${ }^{13,30}$ In particular, the strongest adsorption of $\mathrm{Ti}$ could be attributed to the open $d$ shells in the Ti electronic structure. In the present work, we investigated the electronic structures of selected geometries of CNTs with Pd and Ti nanoparticles and identified that the strongly adsorbed metal particles can induce a strong distortion in the $\pi$ electronic structure of the CNT. In contrast, our previous works showed that the weakly bonded $\mathrm{Al}$ and $\mathrm{Au}$ surfaces give rise to an almost rigid shift in the Fermi level of the CNT, in which the shapes of the density of states are almost intact. ${ }^{31,32}$

We also calculated many adjacent $M_{13}$ particles onto the CNT to simulate the behavior of the accumulated metal

TABLE I. The binding energy $\left[E_{b}(\mathrm{eV})\right]$ of the metal nanocluster $\left(M_{13}\right)$ to the surface of the $(5,5)$ CNT.

\begin{tabular}{lccccc}
\hline \hline & $\mathrm{Al}$ & $\mathrm{Au}$ & $\mathrm{Pt}$ & $\mathrm{Pd}$ & $\mathrm{Ti}$ \\
\hline LDA & 0.39 & 1.24 & 4.45 & 5.49 & 6.53 \\
GGA & 0.04 & 0.25 & 1.73 & 2.60 & 3.71 \\
& & & &
\end{tabular}

(a)
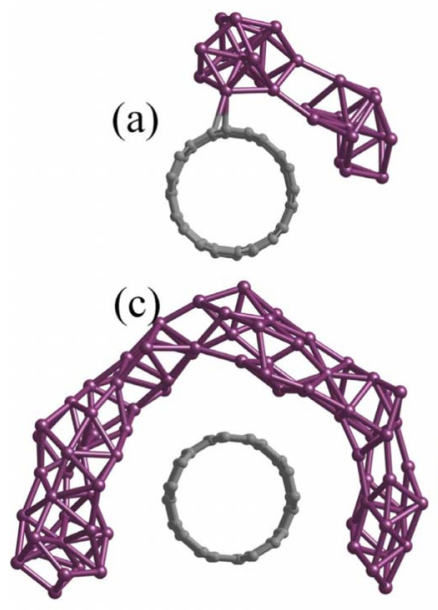

(b)

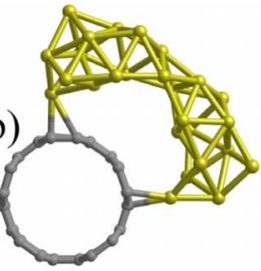

(d)

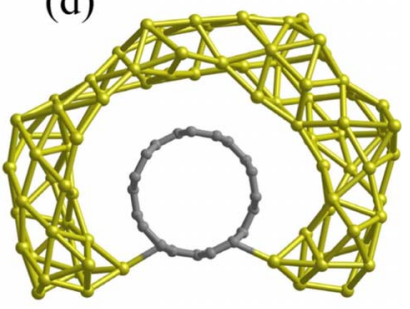

FIG. 2. (Color online) Optimized adsorption geometries of two neighbored (a) $\mathrm{Al}_{13}$ and (b) $\mathrm{Au}_{13}$ particles and six neighbored (c) $\mathrm{Al}_{13}$ and (d) $\mathrm{Au}_{13}$ particles onto the outer wall of the $(5,5) \mathrm{CNT}$.

nanoparticles. The GGA optimized geometries for the cases of two adjacent $\mathrm{Al}_{13}$ and $\mathrm{Au}_{13}$ particles are shown in Figs. 2(a) and 2(b). The very characteristic feature in the present results is that the metal-carbon bond is not favored in these cases. After the geometry optimization, $\mathrm{Al}$ and $\mathrm{Au}$ particles develop a triangular-shape bonding network at the interface with the CNT, resembling the (111) surface. Besides the edge atoms, the $\mathrm{Al}$ and $\mathrm{Au}$ atoms are separated from the CNT surface, as shown in Figs. 2(a) and 2(b). We continued the calculation with six $\mathrm{Al}_{13}$ and $\mathrm{Au}_{13}$ particles surrounding the CNT, as presented in Figs. 2(c) and 2(d). Consistently, the Al and $\mathrm{Au}$ atoms develop a (111)-like triangular network separated from the CNT surface by more than $3.5 \AA$. With the same strategy we investigated the equilibrium geometry of the accumulated Pt, Pd, and Ti particles onto the CNT. We found that they have a good wetting and are well dispersed over the CNT surface, as shown in Fig. 3. Previously, Zhang et al. ${ }^{9}$ and Zhang and Dai ${ }^{10}$ showed that the bare CNT surface could be uniformly coated with $\mathrm{Pd}$ and $\mathrm{Ti}$, whereas the deposited $\mathrm{Al}$ and $\mathrm{Au}$ particles aggregate themselves. Results (a)
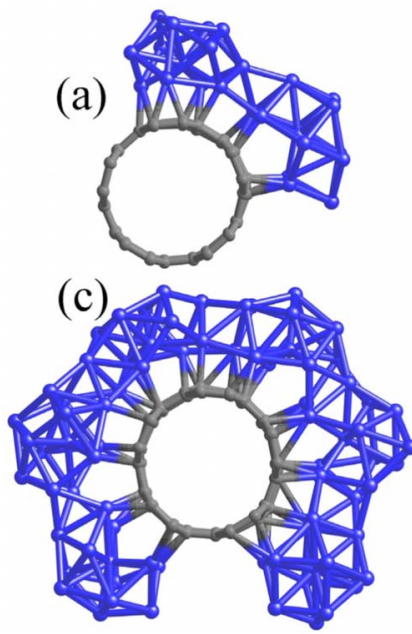

(b)
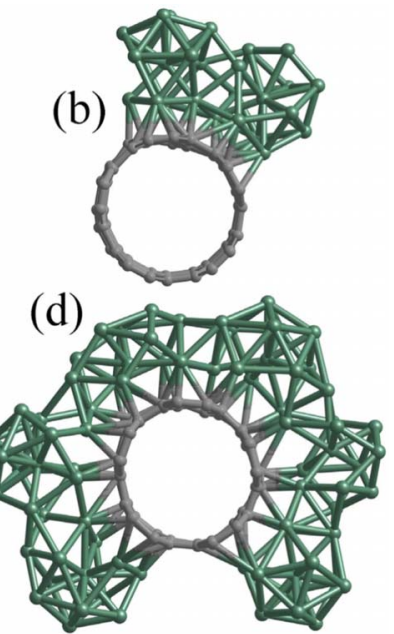

FIG. 3. (Color online) Optimized adsorption geometries of two neighbored (a) $\operatorname{Pd}_{13}$ and (b) $\mathrm{Ti}_{13}$ particles and six neighbored (c) $\operatorname{Pd}_{13}$ and (d) $\mathrm{Ti}_{13}$ particles onto the outer wall of the $(5,5) \mathrm{CNT}$ 
throughout Figs. 1-3 consistently tell that the difference in the wetting properties should be attributed to the difference in the adsorption energetics of the metal nanoparticles with CNTs.

While metallic CNTs have been investigated in view of interconnects, numerous researchers have studied FET-like switchable devices using semiconducting CNTs. ${ }^{3,33}$ One of the principal concerns in those studies is the contact resistance at the metal-CNT interface. Commonly, the Schottky barrier at the interface has been interpreted with the work function of the metal electrodes. ${ }^{16,17}$ However, results of the present work suggest that the local chemistry should be considered as another key factor in determining the overall contact resistance. Note that even though $\mathrm{Au}$ and Pd have very similar work function, their wetting properties are fundamentally different. ${ }^{34}$ As we discussed previously, the Schottky barrier at the metal-CNT interface can vary depending on the bonding strength at the metal-CNT interface. ${ }^{32,35}$ We now understand that the known vulnerability of the Al-CNT contact to oxidation-induced damage is not only due to the strong oxidation tendency of $\mathrm{Al}$ but the weak binding affinity of Al with CNTs. ${ }^{11,12}$ Our results in the present work suggest that the accumulated $\mathrm{Al}$ particles have many voids at the interface with the CNT, and thus oxygen impurities could diffuse into the interface without significant barriers, resulting in the oxidation-induced damage.

In conclusion, we used the $a b$ initio density-functional computational method to investigate the binding affinity and realistic interfacial structures of metal particles onto CNTs. We found that the accumulated $\mathrm{Al}$ and $\mathrm{Au}$ particles develop (111)-like surface at the interface with the CNT in which the metal-carbon bond is energetically unfavorable. Meanwhile $\mathrm{Pt}, \mathrm{Pd}$, and Ti nanoparticles were found to have a decent adsorption strength and a good wetting property. We suggest that a method to increase the binding strength between $\mathrm{Al}$ layer and CNT is required to stabilize the $\mathrm{Al}$ electrode against the oxidation-induced damage.

This research was supported by the Graduate Research Assistantship of Dankook University. N.P. appreciates the support from Samsung Electronics and the TND national project. D.S. and S.H. are supported by the SRC Program (Center for Nanotubes and Nanostructured Composites R112001-091-00000-0) of MEST/KOSEF and by Korea Research Foundation Grant (KRF-2005-070-C00054). Calculations were performed by using the supercomputing resources of KISTI.
${ }^{1}$ C. T. White and T. N. Todorov, Nature (London) 393, 240 (1998).

${ }^{2}$ S. Frank, P. Poncharal, Z. L. Wang, and W. A. De Heer, Science 280, 1744 (1998)

${ }^{3}$ R. Martel, T. Schmidt, H. R. Shea, T. Hertel, and Ph. Avouris, Appl. Phys. Lett. 73, 2447 (1998)

${ }^{4}$ K. A. Dean and B. R. Chalamala, Appl. Phys. Lett. 76, 375 (2000).

${ }^{5}$ T. Rueckes, K. Kim, E. Joselevich, G. Y. Tseng, C.-L. Cheung, and C. M. Lieber, Science 289, 94 (2000).

${ }^{6}$ S. Moon, W. Song, N. Kim, J. S. Lee, P. S. Na, S.-G. Lee, J. Park, M.-H. Jung, H.-W. Lee, K. Kang, and J. Kim, Nanotechnology 18, 235201 (2007).

${ }^{7}$ Z. Yao, C. L. Kane, and C. Dekker, Phys. Rev. Lett. 84, 2941 (2000).

${ }^{8}$ B. Lim, C. Kim, B. Kim, U. Shim, S. Oh, B. Sung, J. Choi, and S. Baik, Nanotechnology 17, 5759 (2006).

${ }^{9}$ Y. Zhang, N. W. Franklin, R. J. Chen, and H. Dai, Chem. Phys. Lett. 331, 35 (2000)

${ }^{10}$ Y. Zhang and H. Dai, Appl. Phys. Lett. 77, 3015 (2000).

${ }^{11}$ H.-S. Kim, B.-K. Kim, J.-J. Kim, J.-O. Lee, and N. Park, Appl. Phys. Lett. 91, 153113 (2007).

${ }^{12}$ B.-K. Kim, N. Park, P. S. Na, H.-M. So, J.-J. Kim, H. Kim, K.-J. Kong, H. Chang, B.-H. Ryu, Y. Choi, and J.-O. Lee, Nanotechnology 17, 496 (2006)

${ }^{13}$ E. Durgun, S. Dag, V. M. K. Bagci, O. Gülseren, T. Yildirim, and S. Ciraci, Phys. Rev. B 67, 201401 (2003).

${ }^{14}$ S. Okada and A. Oshiyama, Phys. Rev. Lett. 95, 206804 (2005).

${ }^{15}$ Z. Chen, J. Appenzeller, J. Knoch, Y.-M. Lin, and Ph. Avouris, Nano Lett. 5, 1497 (2005)

${ }^{16}$ S. Heinze, J. Tersoff, R. Martel, V. Derycke, J. Appenzeller, and $\mathrm{Ph}$. Avouris, Phys. Rev. Lett. 89, 106801 (2002).

${ }^{17}$ V. Derycke, R. Martel, J. Appenzeller, and Ph. Avouris, Appl. Phys. Lett. 80, 2773 (2002).

${ }^{18}$ T. P. Martin, T. Bergmann, H. Göhlich, and T. Lange, J. Phys. Chem. 95, 6421 (1991).

${ }^{19}$ W. Kohn and L. J. Sham, Phys. Rev. 140, A1133 (1965)

${ }^{20}$ G. Kresse and J. Furthmüller, Phys. Rev. B 54, 11169 (1996).

${ }^{21}$ G. Kresse and J. Furthmüller, Comput. Mater. Sci. 6, 15 (1996).

${ }^{22}$ J. P. Perdew, K. Burke, and M. Ernzerhof, Phys. Rev. Lett. 77, 3865 (1996).

${ }^{23}$ D. M. Ceperley and B. J. Alder, Phys. Rev. Lett. 45, 566 (1980).

${ }^{24}$ J. Ihm, A. Zunger, and M. L. Cohen, J. Phys. C 12, 4409 (1979).

${ }^{25}$ M. Böyükata and Z. B. Güvenc, Braz. J. Phys. 36, 720 (2006).

${ }^{26}$ B. Soulé de Bas, M. J. Ford, and M. B. Cortie, J. Phys.: Condens. Matter 18, 55 (2006)

${ }^{27}$ S. H. Yang, D. A. Drabold, J. B. Adams, P. Ordejón, and K. Glassford, J. Phys.: Condens. Matter 9, L39 (1997)

${ }^{28}$ S.-Y. Wang, W. Duan, D.-L. Zhao, and C.-Y. Wang, Phys. Rev. B 65, 165424 (2002).

${ }^{29}$ Q. Zhao, M. B. Nardelli, W. Lu, and J. Bernholc, Nano Lett. 5, 847 (2005).

${ }^{30}$ A. Maiti and A. Ricca, Chem. Phys. Lett. 395, 7 (2004).

${ }^{31}$ N. Park, D. Kang, S. Hong, and S. Han, Appl. Phys. Lett. 87, 013112 (2005).

${ }^{32}$ N. Park and S. Hong, Phys. Rev. B 72, 045408 (2005).

${ }^{33}$ S. J. Tans, A. R. M. Verschueren, and C. Dekker, Nature (London) 393, 49 (1998).

${ }^{34}$ H. B. Michaelson, J. Appl. Phys. 48, 4729 (1977)

${ }^{35}$ S. Moon, S.-G. Lee, W. Song, J. S. Lee, N. Kim, J. Kim, and N. Park, Appl. Phys. Lett. 90, 092113 (2007) 